\title{
Design on the platform system of well-site information center based on WITSML
}

\author{
Qi Zhong ${ }^{1, a}$, Hong $\mathrm{Du}^{2, \mathrm{~b},{ }^{*}}$ \\ ${ }^{1}$ College of Electronic and Information, Yangtze University, Jingzhou 434023, Hubei,China \\ ${ }^{2}$ College of Electronic and Information, Yangtze University, Jingzhou 434023,Hubei,China \\ a email: 297733888@qq.com, ${ }^{\text {b }}$ email: duhmail@126.com
}

\section{Keywords: WITS; WITSML; POSC; Drilling Engineering; Well Site Information Center}

\begin{abstract}
In the drilling site some petroleum technology service companies need to work together in order to complete the whole drilling task, and sometimes they also need technical support from base technical experts, so it is necessary to realize data exchange and data share between the well site and the base. This paper introduces the WITS and WITSML protocol standard, then discusses the system structure, the composition of well site information center platform and data flow, finally introduces the data processing and applications of information center system. The solution described by the paper has been applied in the actual system. It is proved that the solution can solve the problem of system integration in well site, make multiple service companies more smooth collaborative work, and ensure the safety of drilling engineering.
\end{abstract}

\section{Introduction}

Modern oil and gas drilling process is a highly complicated, professional and dangerous project. Therefore, drilling project is experiencing a significant revolution: In the 1980s, companies like Amoco, Mobil, Tennceo and superior began transmitting drilling/logging data remotely and establishing an oilfield data center. In the 1990s, Halliburton Company stared constructing a solution center for exploring and developing real-time oil deposit remotely. At the start of this century, BP developed exploration operation of Gulf of Mexico and set up a visual center of decision and command, and some Chinese companies like Shenkai, Jpsoft also began transmitting drilling/logging data remotely so that drilling experts can examine the situation of drilling directly through network on the oil base.

With the constant improvement of computer and network technology, the information processing and drilling process monitoring of the drilling well-site can make the well site and the oil base keep communication through network. At the same time, it can make a real-time collection of relevant data for experts on the reservoir, drilling project, geology, and well logging of the base to observe the scene, make corresponding decision and direct the operation of drilling engineers on site, thus carrying out the research of information system of the drilling well site to form a comprehensive platform which integrates various professional systems. The acquisition, transmission, storage, demonstration and release of information become the need of data processing and analysis of modern drilling well site[1].

\section{The Standard Protocols for Exchanging Data of Well site}

During the process of well site operations, there are various kinds of equipments which work cooperatively and complete drilling engineering together. In the exploration of the standardization of well site data, WITS (Well site Information Transfer Specification) was put forward to achieve frequent data exchange in the oil exploration and development in the 1980s. WITS is a data wireline communication technology, which can transmit a variety of well site data from one computer system to another, thus forming an industry standard in data sharing and remote monitoring on oil drilling.

The predecessor of Energistics was formed in October 1990 by five founding sponsor oil 
companies: BP, Chevron, Elf, Mobil, and Texaco under the name Petrotechnical Open Software Corporation (POSC-Petrotechnical Open Standards Consortium), and later nearly hundreds of oil and gas companies, service companies and information technology companies including IBM、 SUN、ORACLE、HP、Schlumberger joined them, and worked out a set of industry standards for oil exploration and development application software-SIP ( Open System Software Integration Platform). POSC defines a set of complete open system standards including OS service, communication service, user interaction services, and so on, it provides standards for oil exploration and softeware integration platform development, and petroleum upstream industries adopt POSC integration platform standards by software as WellLogML, LogGraphicsML, ProductionML, XML Components and Objects [1].

With the extensive application of the Internet, WITS data exchange shows a lot of deficiencies. It conducts data transmission point to point, and adopts binary system and ASCII format, but W3C(World Wide Web Consortium) takes XML as the standard of information exchange, which is easy to encode information and is convenient to transmit data among application softwares. Therefore, in October 2000, BP and Statoil and some major service companies initiated and proposed WITSML(Well site Information Transfer Standard Markup Language). WITSML standard evolved from WITS, integrated modern data representative standard XML( Extensible Markup Language) and the Internet standards (W3C, SOAP, XML, WSDL) and established a unified XML format standard achieving effective transmission of drilling data between the well site and the oil base. It could be simply understood as WITSML=WITS+XML [2].

WITSML standard contains two parts in independent versions: WITSML Data Schema and WITSML API [2] [3]. WITSML Data Schema is used to define the content of an XML document including a series of independent and interconnected data object schema. WITSML stipulate 25 standard data records and other 74 user defined records which covers relevant data of drilling and formation evaluation like regular drilling data, surface well-logging, exploration, logging during drilling, communication, drilling machine, drilling fluid system, and directional drilling system. (2) WITSML API defines high-level protocol of data exchanging dynamic program between computer system based on W3C Web communication standard which contains two types of interfaces: Store Interface and Publish Interface.

\section{The Architecture of Well site Information Platform}

In recent years, the concept "digital oilfield” has already formed. Part of the foundation of digital oilfield is a series of operations aimed at well-site data, for example, collection, conversion, transmission, storage and application. In order to achieve optimizing exploration and development of oil and gas, it is necessary to optimize hardware and software, which is the embodiment of information center platform of well site. The platform provides seamless linking for different equipments which internal data format are imcompatible, and carries out information sharing and exchange of equipments or software between the well site service companies or service company and the oil company in oil base[4].

Firstly, well site information system acquires all kinds of project signal from sensors fixed on the drilling rig, the data acquisition modules transfers the data to acquisition computer by CAN bus, the professional software calculates into physical parameters from the data, and stores it into real-time database in the well site. The other applications uses the data to monitor and alarm the drilling. At the same time, the data is transmitted to oil base global database for permanently storage.

Oil field information center converges thousands of well site terminal information for transmision, processing and storage. Oilfield base set up a unified professional center of production management, command and decision, and the relevant managerial or technical personnel can publish data which use these global databases by web or professional software to provide omnidirectional production coordination, technical support and guidance, accident disposal and important command decision-making for each drilling well site of the entire oil field.

In the system, collected well site data are various. The data might derive from comprehensive 
logging instruments, logging while drilling or measurements-while-drilling tools, drilling parameters instrument and geosteering equipment. Then, formats of data are also various. There are three ways for data integration: (1) receiving standard WITS and WITSML protocol data, (2) analysing network packages of transport format formulated by some company, (3) extracting data from the tables of real-time database. Moreover, the network environment of transmitting data is various as well. The transmission modes might be mobile communication network, wireless communication network (radio station, wireless bridge), satellite communication or VPN of Internet. Therefore, the well site information center is a complete system of integrating acquisition, transmission, storage, processing and application. The system structure of the well site information center is shown in Figure 1.

\section{Data Processing}

The appearing of WITSML provides a good petroleum industry standard between the well site and the oil base, which enables the service company of the well site and the application software system of the base to achieve seamless connection without considering the sources of data, thus forming an integrated information platform system between the well site and the oil base[4]. Its data flow diagram is seen in Figure 2.

Firstly, WITSML can receive from different data which might be obtained from multiple software suppliers, or from all kinds of data of the well site, such as geo-steering, measuring while drilling, well logging during drilling, formation pressure prediction and oil testing. With the development of new technologies, more and more data are collected, stored temporarily, used in the well site or sent back to the site information center. through communication channels. The collection methods has turned from simple local data integration into the information platform between the well sites and the oil base that supports local collection, storage, using as well as off-site data collection and integrating.

Secondly, WITSML can convert all types of data into a unified WITSML format by oil data conversion standard. The conversion standardize the data of the drilling well, and XML Data Schema records drilling data, formation evaluation data and irregular data in a dependent and interconnected date object set, which could be transmitted in XML document. After conversion, the extensive well site data all take WITSML XML document as standard data format, which significantly improves development efficiency of data applications at later stages, and saves costs.

Thirdly, WITSML data loading technique, by connecting to WITSML server with WISML application interface, loads local data (trajectory data, MWD/LWD data etc) into global project database of the oil base, accomplishes data transference and entire storage, and provides such functions as data storage, data access of client applications and data publishing.

Fourthly, technicians effectively utilize the data of project database to develop all kinds of web applications that comply with the data exchange capacity of WITSML format, and use XML technique to define data format and apply SOAP protocol that conforms to W3C standard to exchange data, which enables geologists and engineers of the base to manipulate familiar application interface to detect and analyze data in real time, and remotely monitor on-site equipments to achieve application of well site data by network. 

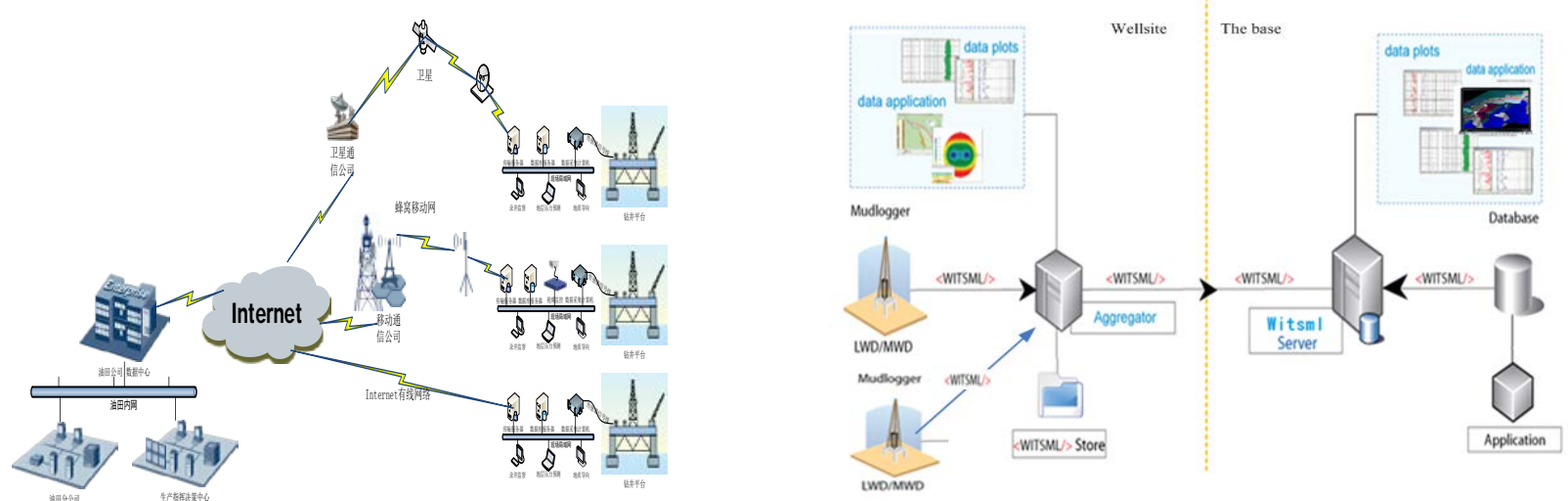

Figure 1 Architecture of the Well site Information Center Figure 2 WITSML Data Flow Diagram

\section{Application of drilling engineering}

Such a well site information center is associated with the expert system of the base and the system of decision and support. The use of conclusions or plans of the expert system and the system of decision and support makes the on-site operation possible[5]. Under this circumstance, one function of the well site information center is the performance of base operations which send the data (state) of well site to the base, visualize WITSML data through some application softwares and provide data to the third party system for real-time analysis, or provide experienced experts or decision and support system on the base to make judgements and solutions so as to control well-site softwares or equipments to adjust wellsite drilling construction.

\section{Conclusion}

WITSML has already become the international standard of data exchange for oil drilling engineering now. We constructs the drilling information center platform by kinds of communication network using WITSML protocol standard , it connects well sites and the oil base. We believe that with the increase of WITSML system softwares, the application of WITSML would extend constantly, and guarantee the real-time processing of field data and the comprehensive application of base data, thus promoting the development of digital oil field, saving costs and increasing efficiency.

\section{References}

[1] Study on POSC Technical Report, http://doc.mbalib.com/view/71b536ee8d4a

[2] http://www.witsml.org/

[3] http://www.energistics.org/witsml-standard

[4]M.A Kirkman,SPE,BP;M.E.Symmonds,SPE,Schlumberger...,Wellsite Information Transfer Standard Markup Language (WITSML) an Update.

[5]Zhongfei Ren, Baorong Zhong, WITSML standard and its application, Computer application in petroleum industry [J], 2012(1) 9-11. 